\title{
A Cross-Device Spatial Workspace Supporting Artifact-Mediated Collaboration in Interaction Design
}

\section{Florian Geyer}

University of Konstanz

Human-Computer Interaction Group

Universitätsstrasse $10, \mathrm{BO} \times \mathrm{D} 73$

DE 78457 Konstanz, Germany

Florian.Geyer@uni-konstanz.de

\section{Harald Reiterer}

University of Konstanz

Human-Computer Interaction Group

Universitätsstrasse 10, Box D 73

DE 78457 Konstanz, Germany

Harald.Reiterer@uni-konstanz.de

\begin{abstract}
In this paper we present our approach to support artifact-mediated collaboration in interaction design. We argue that the extensive number and the diversity of artifacts created and reflected upon during collaborative design activities as well as transitions between physical and digital representations impose both a challenge and opportunity for supporting interaction design practice. The design principles for our experimental tool that we introduce within this paper are based on a cross-device spatial workspace synchronized by a shared design artifact repository within a computation-augmented design studio setting.
\end{abstract}

\section{Keywords}

Artifact-mediated Collaboration, Design Tools, Design Process, Design Artifact Visualization

\section{ACM Classification Keywords}

H.5.3 [Information Interfaces and Presentation]: Group and Organization Interfaces - Collaborative Computing

\section{General Terms}

Design, Experimentation 


\section{Introduction}

Cognition has traditionally been regarded as the outcome of internal cognitive processes manipulating mental representations [9]. However, it has been shown [6] that cognition cannot be separated from the material and social environment. In many cases, like collaborative design, cognition is distributed among environmental properties and individuals. Social interactions between designers are often accompanied by indirect communication via artifacts for high-level activities like coordination and group reflection [8].

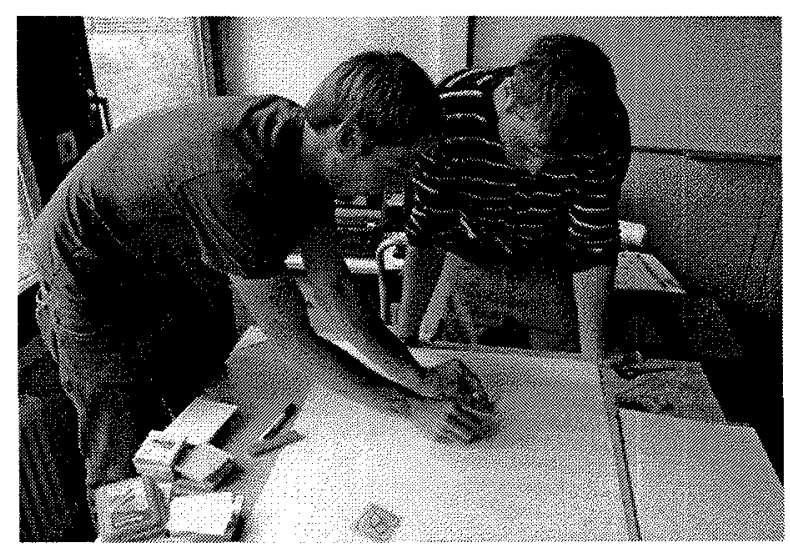

figure 1. Interacting with physical artifacts during

collaborative ideation activities. Note how the nature of material artifacts facilitates group interaction but also makes them hard to share and archive.

Hence, during design activities physical artifacts like sketches and pictures are used as a basis for both individúal and collaborative creative work. They provide representations for organizing discussion, serve as reference material throughout the design process and extend the designer's psychological abilities like

remembering, self-assessment, associating and judging [2]. When interacting with artifacts - e.g. in design meetings - the properties of material representations allow to view, to gesture on, to navigate between and to annotate them [9]. However, it has been observed [11] that bottlenecks are created when digital artifacts are used during the design process as their

representations often do not facilitate or invite these interactions.

\section{Artifacts in Interaction Design}

In interaction design practice a variety of artifacts are used as a tool for inspiration, individual reflection and for communicating ideas within a group [2,3]. Within ideation activities, physical artifacts like post-its, pictures, paper sketches, sketch books and whiteboards are often preferred due to their flexibility, unique affordances and accessibility [11]. In design-critique, material artifacts are usually presented to others in studio-like environments on pin boards or display walls $[2,3,10]$. However, the extensive number of artifacts and their physical nature impose additional efforts for sharing and archiving them in digital repositories which is often required over the course of a design project or across different design projects.

Nevertheless, digital artifacts like videos, websites, digital images or prototype simulations also serve as important sources of inspiration. While these artifacts are capable of representing dynamics, enable rich interactions and are easy to share and archive, they do not have the unique affordances of physical artifacts. Digital artifacts are frequently hidden in file systems and therefore hardly visible and accessible within group 
work. Bodily and social factors that are crucial for reflective design practice $[4,10]$ are often mistreated.

\section{Tool Support}

We argue that in order to effectively support artifactmediated collaboration in interaction design it is crucial to provide adequate tool support. Digital artifacts are often tied to specific tools that are used to create, to refine or to share them. While these tools bring the benefits of digital information processing and rich dynamics, they often do not have the affordances of material artifacts. Due to this duality of representation and the different qualities for designers, transitions between physical and digital artifacts and vice versa are quite common. As a result of these transitions the overall flow of the design process is harmed. We think that traditional desktop-based tools seem not to be capable of providing the required qualities for artifactmediated collaboration since they are often built for specific tasks like image manipulation, video editing sketching or prototyping instead for the overall activity of designing. In contrast, physical artifacts are more versatile and allow for flexible use over different collaborative design activities. For example, post-its are frequently used tools across different tasks like paper prototyping, stop-motion animation or affinity

diagramming [3]. Similar flexibility is yet unmatched with digital tools. However, beyond-the-desktop technologies like multi-touch tabletops, digital pen \& paper as well as interactive whiteboards may provide opportunities for more adequate tool support.

The need for more flexible design tools is well recognized within the $\mathrm{HCI}$ community. Researchers work on bringing interactions with digital tools closer to physical practice. Tools were introduced that integrate pen input and different modalities to facilitate sketching and annotations in group sessions [5]. Efforts to improve accessibility of digital artifacts for co-located collaboration like cross-device workspaces $[1,12]$ as well as groupware for design [13] are promising. It was also shown with tools like Cabinet ${ }^{1}$ and Outpost [7] that combinations of digital technology with material artifacts can make collaborative design more tangible. While many of these approaches focus on technical infrastructure or are specific to certain tasks, we argue that an activity-centered approach that is based on basic requirements for artifact-mediated collaboration can be successful in a variety of design activities.

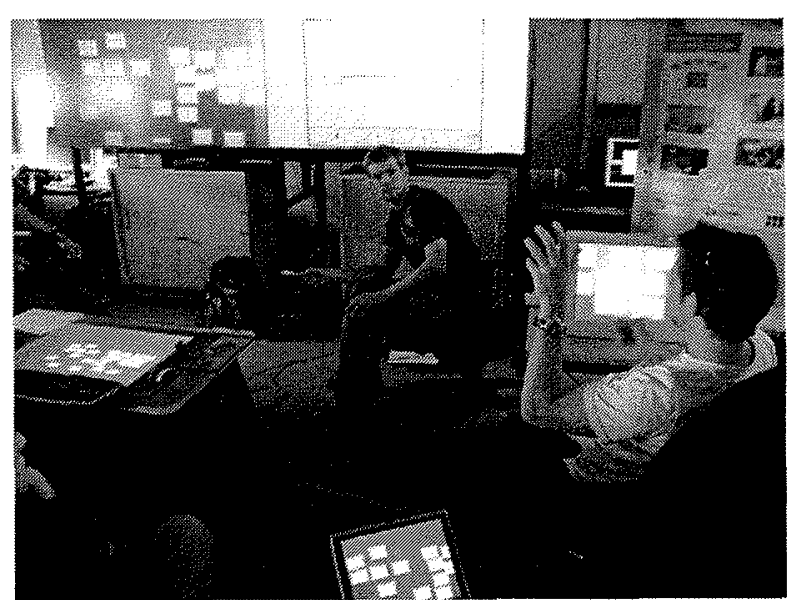

figure 2. Beyond-the-desktop technologies may provide the infrastructure for supporting the physical, cognitive and social aspects of artifact-mediated collaboration.

1 http://studiolab.io.tudelft.nl/cabinet/ 


\section{Our Approach}

We think that collaborative design is an interplay of physical, cognitive and social factors. Therefore, we aim at supporting these aspects for the overall activity of designing instead of focusing on specific tasks. We seek to bring the most important qualities of physical artifacts to digital tools by augmenting artifacts with technology and by extending digital artifacts with

physical affordances and flexibility of use. The resulting benefits may include improved artifact management techniques based on digital repositories, flexibility and reuse of digital artifacts as well as reduced costs of transitioning between physical and digital

representations. We have four design principles for creating such a tool that we describe in the following.

Shared workspace as virtual pin board

We think that digital repositories and databases can provide an adequate technical infrastructure to share artifacts within design teams. They can improve accessibility of artifacts and allow archiving them for reuse. However, instead of hiding artifacts within spreadsheets or websites, the repository should be accessible via adequate visualizations that closely resemble physical practice. We explore the metaphor of a virtual pin board where team members can create and share artifacts as an interface to the repository which is implemented as an object-oriented database that is capable of synchronizing attached clients.

Spatial visualization of artifacts

One of the key characteristic of material artifacts is spatial positioning. By leveraging the ability to physically align, juxtapose and compare different artifacts in space designers harness their spatial thinking abilities [14]. This is especially important within individual and collaborative reflection and synthesis phases [4]. Within our virtual pin board we explore spatial arrangements that support designers in these tasks over a range of activities throughout the process (e.g. timelines, process maps, affinity diagrams). We believe that integrating a variety of artifacts from sketches, pictures and videos or even prototype simulations into our interactive visualizations will improve flexibility of use. As the number of artifacts may become extensive, we integrate advanced zooming techniques to navigate on the virtual pin board.

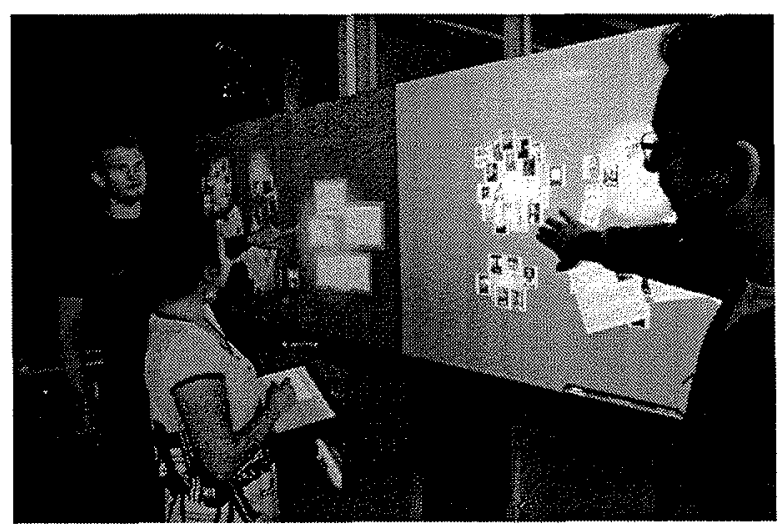

figure 3. Spatial positioning and scaling of artifacts on a zoomable pin board allows for different arrangements like timelines or process maps for cooperative reasoning. The visualization on an interactive whiteboard allows viewing, navigating, annotating and gesturing on artifacts.

Cross-device interaction

As artifacts need to be accessible by multiple persons at the same time to facilitate efficient collaboration, we 
build our tool around a physical setting similar to a design studio (see figure 2). As one important characteristic of a real design studio is physical display space, our computation-augmented design studio features several large, high-resolution interactive displays in the form of two tabletops and one large interactive whiteboard with pen interaction. For individual work, we integrated graphic tablets and tablet PCs.

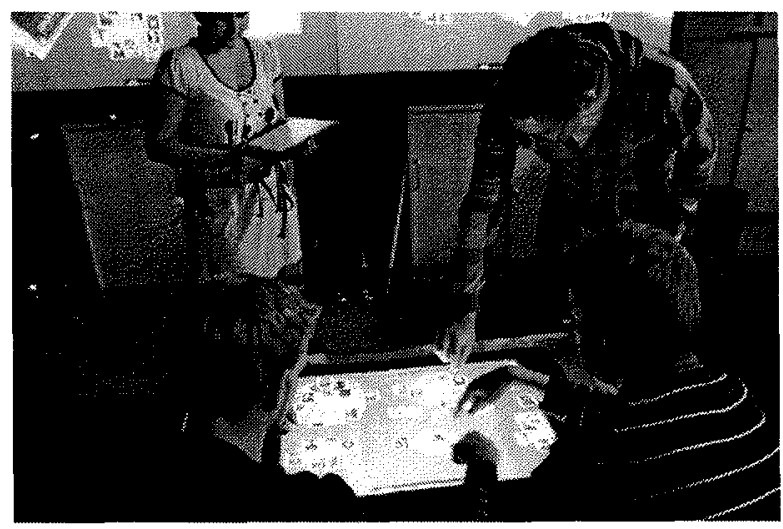

figure 4. Interacting with digital artifacts on an interactive tabletop. The affordances of multi-touch technology invite similar interactions as material representations and provide a hands-on experience when sorting, aligning or scaling artifacts.

Each device allows an independed view on the artifacts that are stored in the repository. However,

manipulations like positioning and annotating are synchronized over the repository. The zoomable interface of the virtual pin board allows navigating between clusters and individual artifacts to focus on specific aspects while still preserving the context. We are attempting to design the interface in a consistent way that works across different devices and screen sizes. We also aim at designing intuitive cross-device interaction techniques.

Physical affordances

As digital artifacts on the virtual pin board lack physical affordances, we are designing their representation with the goal to reproduce some of the physical attributes.

By facilitating multi-touch input on our tabletops and

tablet PCs, we allow hands-on experiences when

sorting, aligning and scaling artifacts. Physical

attributes are also imitated by naïve physic effects that furthermore stimulate a playful interaction.

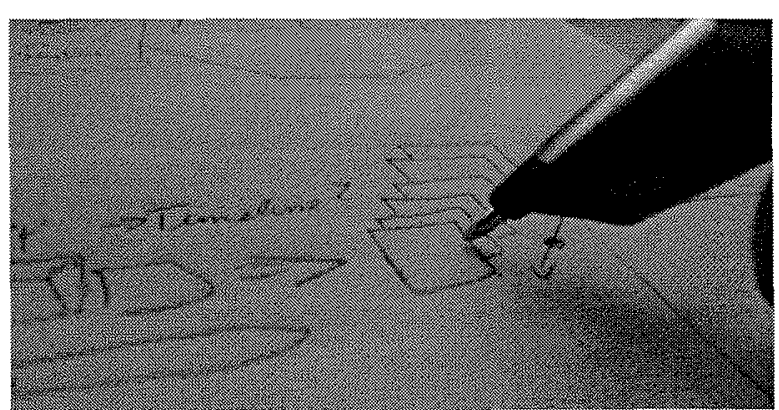

figure 5. Digital pen \& paper technology allows to integrate physical paper into our cross-device workspace. Artifacts can be instantly transferred from digital to material representation and vice versa.

Sketches and annotations can be created with digital pens on the whiteboard, graphic tablet and on tablet PCs. However, we think that stylus interaction does not have the same qualities as writing on real paper.

Therefore, we integrated digital pen \& paper technology 
from Anoto ${ }^{2}$. This technology enables us to create or refine artifacts on real paper by still allowing instant digital access via the repository. Furthermore, digital artifacts can be printed on physical paper for

distribution and annotation in group discussions. We think that the tangible nature of these interactive paper artifacts can further improve flexibility of use across different design activities.

\section{Conclusion}

We presented our motivation and approach for supporting artifact-mediated collaboration techniques in interaction design practice. In future work we aim at developing techniques that harness the basic concept of our cross-device spatial workspace over a variety of design activities. We believe that integrating existing artifact-centric techniques into our workspace will bring valuable insights for improving tool support for highlevel design activities.

\section{Acknowledgements}

This research is funded by the German Research

Foundation DFG (Deutsche Forschungsgemeinschaft) under grant number RE 1843/3-1.

\section{References}

(1] Borchers J., Ringel M., Tyler J., Fox A.: Stanford Interactive Workspaces: A Framework for Physical and Graphical User Interface Prototyping. Wireless Communications, IEEE (2002), 64-69.

[2] Brown J., Lindgaard G., Biddle R.: Stories, Sketches, and Lists: Developers and Interaction Designers Interacting Through Artefacts. Proceedings of AGILE Conference, IEEE (2008), 39-50.

${ }^{2}$ www.anoto.com
[3] Buxton, B.: Sketching User Experiences: Getting the Design Right and the Right Design. Morgan Kaufmann (2007).

[4] Cross, N.: Designerly Ways of Knowing. Board of International Research in Design. Birkhäuser (2007).

[5] Hailpern J., Hinterbichler E., Leppert C., Cook D., Bailey B. P.: TEAM STORM: Demonstrating an

Interaction Model for Working with Multiple Ideas

During Creative Group Work. Proceedings of Creativity \& Cognition, ACM (2007), 193-202.

[6] Hutchins E.: Cognition in the Wild. MIT Press (1995).

[7] Klemmer S. R., Newman M. W., Farrell R., et al.: The Designers' Outpost: A Tangible Interface for Collaborative Web Site Design. Proceedings of UIST, ACM (2001), 1-10.

[8] Perry M., Sanderson D.: Coordinating Joint Design Work: The Role of Communication and Artefacts. Design Studies, Elsevier (1998), 273-288.

[9] Rambusch J., Susi T., Ziemke T.: Artefacts as Mediators of Distributed Social Cognition: A Case Study, Proceedings of Social Cognition, Lawrence Erlbaum (2003), 1113-1118.

[10] Schön, D. A.: The Reflective Practitioner: How Professionals Think in Action. Basic Books (1983). [11] Sellen A., Harper R.: The Myth of the Paperless Office. MIT Press (2001).

[12] Streitz N. A., GeiBler J., Holmer T., et al.: i-LAND: An Interactive Landscape for Creativity and Innovation. Proceedings of CHI, ACM (1999), 120-127.

[13] Venkataswamy A., Sodhi R., Abdildin Y., Bailey B. P.: Groupware for Design: An Interactive System to Facilitate Creative Processes in Team Design Work. HICSS, IEEE (2009), 1-10.

[14] Vyas D., Veer G.V., Heylen D., Nijholt A.: Space as a Resource in Creative Design Practices. Proceedings of INTERACT 2009, Springer (2009), 169-172. 